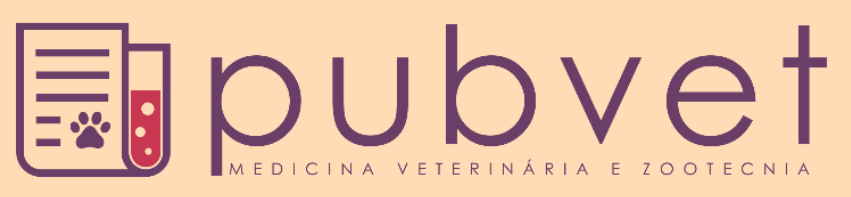

https://doi.org/10.31533/pubvet.v15n03a772.1-7

\title{
Avaliação do efeito analgésico do maropitant em gatas submetidas à ovariohisterectomia eletiva
}

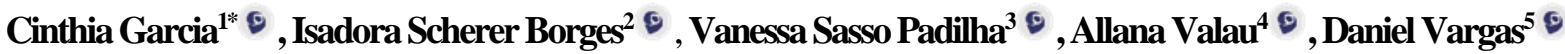 \\ ${ }^{1}$ Médica Veterinária na Clínica Veterinária Animal Center, Criciúma, Santa Catarina, Brasil.E-mail: cinthiagarcia388@gmail.com* \\ ${ }^{2}$ Médica Veterinária, Palmeira das Missões, Rio Grande do Sul, Brasil \\ ${ }^{3}$ Professora da Universidade Federal de Santa Catarina, Curitibanos, Santa Catarina, Brasil. \\ ${ }^{4}$ Professora da Universidade do Oeste de Santa Catarina, Campos Novos, Santa Catarina, Brasil. \\ ${ }^{5}$ Mestrando na Universidade Federal de Santa Maria, Santa Maria, Rio Grande do Sul, Brasil.
}

\begin{abstract}
Resumo. O objetivo deste estudo foi analisar o efeito analgésico do maropitant, comparado a morfina isolada ou associação de ambos os fármacos, quando utilizados como medicação pré-anestésica em procedimento de ovariohisterectomia eletiva na espécie felina. Foram avaliados os parâmetros fisiológicos e plano anestésico de 18 gatas no período transcirúrgico, além do comportamento em 2, 4, 6, 8, 12 e 24 horas pós-cirúrgicas através da Escala Analógica Visual e da Escala Multidimensional da Dor Aguda em Felinos por dois avaliadores cegos ao tratamento. Pode-se concluir que o uso do maropitant isolado ou associado a morfina teve a mesma eficácia analgésica da morfina
\end{abstract}

Palavras chave: Comportamento, dor, felinos

\section{Evaluation of the analgesic effect of maropitant in cats submitted to elective ovariohysterectomy}

\begin{abstract}
The aim of this study was to analyze the analgesic effect of maropitant, compared to isolated morphine or combination of both drugs, when used as pre-anesthetic medication in elective ovariohysterectomy procedure in feline species. The physiological parameters and anesthetic plan of 18 cats in the trans-surgical period were evaluated, in addition to the behavior in 2, 4, 6, 8, 12 and 24 hours post-surgery using the Visual Analogue Scale and the Multidimensional Scale of Acute Pain in Felines for two treatment-blind evaluators. It can be concluded that the use of maropitant alone or associated with morphine had the same analgesic efficacy as morphine.
\end{abstract}

Keywords: Behavior, ache, cats

\section{Evaluación del efecto analgésico de maropitant en gatos sometidos a ovariohisterectomía electiva}

Resumen. El objetivo de este estudio fue analizar el efecto analgésico del maropitant, en comparación con la morfina aislada o la combinación de ambos fármacos, cuando utilizados como medicación preanestésica en un procedimiento de ovariohisterectomía electiva en felinos. Se evaluaron los parámetros fisiológicos y plan anestésico de 18 gatas en el período transquirúrgico, además del comportamiento en 2, 4, 6, 8, 12 y 24 horas postoperatorias utilizando la Escala Visual Analógica y la Escala Multidimensional de Dolor Agudo en Gatos por dos evaluadores ciegos al tratamiento. Se puede concluir que el uso de maropitant solo o asociado a morfina tuvo la misma eficacia analgésica que la morfina.

Palabras clave: Comportamiento, dolor, felinostos 


\section{Introdução}

Embora o cão tenha maior destaque em relação a interação com as pessoas, nos últimos anos, houve um aumento significativo na população de felinos (Anderline, 2007; Beaver, 2005). No Brasil, a população de felinos cresce a taxas maiores que a dos cães.

Em relação à dor na $\mathrm{OSH}$, a reação inflamatória da ovariohisterectomia aumenta a liberação de prostaglandinas que estimulam os nociceptores, tornando-a um procedimento doloroso (Teixeira \& Figueiró, 2001), onde a manipulação de órgãos abdominais contribui para o incremento da dor durante várias horas após a intervenção cirúrgica (Fantoni et al., 2002; Mastrocinque, 2005). Embora o tratamento da dor seja um tópico emergente e popular na medicina veterinária, o uso em gatos é menor em comparação aos cães (Hellebrekers, 2002). Uma das principais causas é provavelmente a dificuldade de reconhecer e tratar a dor nesta espécie (Brondani et al., 2011). Contudo, o médico veterinário e sua equipe tem a responsabilidade de reconhecer, avaliar, prevenir e tratar a dor, através das alterações fisiológicas e comportamentais (Hellyer, 1997; Short, 1998).

Marquez et al. (2015) realizaram o primeiro estudo comparando o uso de morfina ou maropitant, como um pré-anestésico, para OSH em cadelas. Os parâmetros registrados mostraram semelhanças entre os grupos durante o procedimento cirúrgico. Maropitant, por ser um potente antiemético e reduzir a náusea pós-operatória, pode ser parte da explicação de poder ser adequado como um agente préanestésico. Outra hipótese é a capacidade potencial desse fármaco para diminuir a dor visceral, que chega por vias nervosas às estruturas do cérebro envolvidas na nocicepção e no processamento da dor, que contém NK-1 receptores e substância P, no entanto, o sítio específico de ação para os antagonistas do receptor NK-1, como maropitant, requer uma investigação mais aprofundada.

O objetivo deste estudo foi avaliar a analgesia promovida pelo Maropitant, quando administrado como medicação pré-anestésica, isolado ou associado à morfina por meio da avaliação da dor durante o procedimento cirúrgico e pós-operatório em gatas submetidas ao procedimento de ovariohisterectomia eletiva.

\section{Material e métodos}

Dispôs-se de 18 gatas hígidas, dóceis, livres de cio, não prenhes, com idade a partir de 6 meses, sem raça definida. Para a seleção dos animais foi realizado o exame físico e clínico, assim como hemograma, proteína plasmática total, e os bioquímicos, ureia, creatinina, fosfatase alcalina, gama glutamil transferase e alanina aminotransferase, a fim de atestar um bom estado de saúde. 24 horas previamente ao procedimento cirúrgico, os animais foram alojados em sala silenciosa, individualmente, e submetidos a interação com os dois avaliadores (A e B), de forma a ambientar-se com o local. Após os avaliadores analisarem o animal e terem conhecimento de seu comportamento, foi realizada mensuração da pressão arterial sistólica (PAS) basal, e posterior tricotomia da região abdominal e ambas as veias cefálicas, além de jejum sólido e hídrico por 12 e 6 horas, respectivamente.

Subdividiu-se três grupos de seis animais, aleatoriamente, os quais receberam os seguintes tratamentos: maropitant (GMA) $(1 \mathrm{mg} / \mathrm{kg})$, maropitant associado à morfina (GMM) $(1 \mathrm{mg} / \mathrm{kg}$ e 0,3 $\mathrm{mg} / \mathrm{kg}$, respectivamente), ou morfina (GMO) $(0,3 \mathrm{mg} / \mathrm{kg})$, aplicados por via intravenosa. Após 5 minutos, a indução anestésica foi realizada com propofol dose efeito, posteriormente foram intubadas com sonda endotraqueal adequada ao seu porte, e manutenção anestésica com propofol na taxa variável para manutenção do plano ideal, e fornecimento de oxigênio a $100 \%$ com fluxo de $200 \mathrm{~mL} / \mathrm{kg} / \mathrm{min}$, em circuito aberto sem reinalação de gases (Baraka). Todos os animais receberam fluidoterapia com ringer lactato na taxa de $5 \mathrm{~mL} / \mathrm{kg} / \mathrm{h}$. O procedimento cirúrgico de ovariohisterectomia eletiva foi realizado em todos os animais pelo mesmo cirurgião e auxiliar para não ocorrer disparidade na manipulação entre os diferentes animais. Durante o procedimento cirúrgico, foram avaliados os momentos M1 (paciente em plano anestésico); M2 (incisão da musculatura); M3 (após pinçamento do primeiro pedículo); M4 (após pinçamento do segundo pedículo); M5 (após pinçamento da cérvix); M6 (após sutura da musculatura); e M7 (ao final da cirurgia), sendo mensurados parâmetros de frequência cardíaca (FC), saturação de oxihemoglobina (SO2) e frequência respiratória (FR) através do monitor multiparamétrico, temperatura corporal $\left(\mathrm{T}^{\circ} \mathrm{C}\right)$ com o auxílio do termômetro esofágico e pressão arterial sistólica (PAS) com o método de doppler. 
Além disso, foi avaliado o plano anestésico dos animais através dos reflexos oculopalpebrais, corneal e pupilar, classificando-os em superficial (animal apresentando globo ocular centralizado ou rotacionado, com presença de reflexos palpebrais medial e lateral e tônus mandibular), ideal (globo ocular rotacionado, sem presença de reflexos palpebrais ou com presença de reflexo palpebral medial e sem tônus mandibular) ou profundo (globo ocular centralizado, sem reflexos palpebrais nem tônus mandibular). Tempo para a extubação e horário em que o animal assumiu decúbito esternal também foram avaliados. Durante a recuperação examinou-se o animal nos tempos 2 (T2), 4 (T4), 6 (T6), 8 (T8), 12 (T12) e 24 horas (T24) após o procedimento cirúrgico, em que os avaliadores (A e B), sem conhecimento à qual grupo o animal pertencia, pontuaram o comportamento do animal e suas expressões de acordo com a escala analógica visual (EAV) e da escala multidimensional de dor aguda em felinos (EMADOR). $\mathrm{O}$ animal que apresentasse pontuação igual ou maior que 8 , em algum dos momentos da avaliação pela escala EMADOR, e 30\% ou mais pela EAV foi submetido a resgate analgésico com morfina na dose de $0,2 \mathrm{mg} / \mathrm{kg}$ por via intramuscular.

Os dados coletados foram submetidos ao teste de normalidade de Shapiro-Wilk e posteriormente à análise estatística com auxílio do software SigmaStat $12^{\circledR}$. Para os dados paramétricos, a análise entre os grupos dentro de cada momento foi utilizada ANOVA e para análise entre tempos dentro do mesmo grupo, ANOVA de uma Via com Repetições Múltiplas (ANOVA-RM). Para os dados não-paramétricos foi utilizado o teste de Kruskal Wallis para comparação entre os tempos dentro do mesmo grupo, e o teste de Friedmann para comparação entre os grupos. Para análise dos resgates analgésicos foi realizada a curva de Kaplan-Meyer. As diferenças foram consideradas estatisticamente significativas quando $\mathrm{P} \leq 0,05$.

\section{Resultados e discussão}

Os resultados provenientes da estatística demonstraram homogeneidade entre grupos com relação ao peso e exames pré-operatórios (hemograma, leucograma e bioquímicos), pois não houve diferença ( $\mathrm{P}>$ $0.05)$ entre grupos.

Não houve efeito adverso após a aplicação dos fármacos morfina e/ou maropitant pela via intravenosa. Náusea e vômito podem ser ocasionados pela morfina devido à estimulação da zona deflagradora dos quimiorreceptores localizada nas paredes laterais do terceiro ventrículo (área postrema) (Spinosa et al., 2006). Já o maropitant é uma droga com efeito antiemético. No estudo de Swallow et al. (2017), no qual cães receberam o referido fármaco na dose $1 \mathrm{mg} / \mathrm{kg}$ pela via subcutânea, não houve relatos de efeitos adversos após sua aplicação.

No trans-operatório não houve diferenças $(\mathrm{P}>0.05)$ entre grupos em todas as variáveis analisadas, mostrando que os três protocolos promoveram efeitos semelhantes para o procedimento cirúrgico de ovariohisterectomia.

De acordo com a tabela 1 podemos observar as diferenças entre os momentos avaliados dentro do mesmo grupo. As diferenças na FC e PAS podem ser explicadas devido ao fato que no M1 não havia nenhum estímulo sendo aplicado nos pacientes, já nos demais momentos avaliados a cirurgia já estava ocorrendo, o que corrobora com Hellyer et al. (2007), o qual afirma que estímulos álgicos levam a variações nos parâmetros fisiológicos. Ainda, de acordo com Silva (2011), deve-se utilizar parâmetros fisiológicos para avaliação de nocicepção em pacientes anestesiados, como frequência cardíaca e respiratória, pressão arterial e temperatura. Esses parâmetros tendem a aumentar quando há estímulo doloroso, caracterizando uma analgesia inadequada. Podemos observar na tabela 1 que nos momentos M1, M2 e M3 alguns animais apresentaram PAS abaixo de $90 \mathrm{mmHg}$, caracterizando hipotensão. Essa redução da pressão arterial pode ser explicada pelo uso do propofol para indução e manutenção anestésica, ou pela aplicação dos tratamentos, morfina e maropitant.

No estudo de Liehmann et al. (2006), em que gatos foram induzidos com propofol e mantidos com propofol associado a fentanil ou isoflurano associado ao fentanil, ambos os grupos apresentaram quadros de hipotensão, porém no grupo propofol/fentanil a hipotensão foi descrita como leve e de curta duração, o que corrobora com os achados do presente estudo, em que a PAS foi se elevando com o grau de estímulo doloroso aplicado, normalizando e não voltando a reduzir ao final do procedimento. No estudo de Corrêa et al. (2019), em que gatas receberam maropitant na forma de bolus pela via intravenosa seguido de infusão contínua, também foi observado hipotensão, sendo que quanto maior a taxa de 
infusão do maropitant, maior foi a redução da pressão arterial. A substância $\mathrm{P}$ está envolvida na regulação da pressão arterial e frequência cardíaca (Garcia-Recio \& Gascón, 2015), sendo classificada como uma taquicinina. Taquicininas quando aplicadas intracerebroventricular promovem aumento da pressão arterial e frequência cardíaca por aumento da transmissão dopaminérgica (Deschamps \& Couture, 2005); portanto, sendo o maropitant capaz de atravessar a barreira hematoencefálica e bloquear os receptores NK-1 centralmente (de la Puente-Redondo et al., 2007), pode ocasionar queda de pressão arterial e de frequência cardíaca

Tabela 1. Médias e desvio padrão das variáveis de gatas submetidas a ovariohisterectomia, e tratadas com morfina, maropitant ou morfina associada a maropitant

\begin{tabular}{|c|c|c|c|c|c|c|c|c|}
\hline Variáveis & Tratamento & $\begin{array}{l}\text { M1: Paciente em } \\
\text { plano anestésico }\end{array}$ & $\begin{array}{c}\text { M2: Incisão } \\
\text { da } \\
\text { musculatura }\end{array}$ & $\begin{array}{c}\text { M3: Após } \\
\text { pinçamento do } \\
\text { primeiro pedículo }\end{array}$ & $\begin{array}{c}\text { M4: Após } \\
\text { pinçamento do } \\
\text { primeiro pedículo }\end{array}$ & $\begin{array}{c}\text { M5: Após } \\
\text { pinçamento da } \\
\text { cérvix }\end{array}$ & $\begin{array}{l}\text { M6: Após } \\
\text { sutura da } \\
\text { musculatura }\end{array}$ & $\begin{array}{l}\text { M7: Ao final } \\
\text { da cirurgia }\end{array}$ \\
\hline \multirow{3}{*}{ Frequência cardíaca, bpm } & Morfina & $167,0 \pm 17,0 \mathrm{~A}$ & $154,0 \pm 27,2 \mathrm{a}$ & $151,6 \pm 48,7$ & 34,3 & $199,8 \pm 34,4$ & $203,6 \pm 32,7 \mathrm{~B}$ & $209,3 \pm 24,8$ \\
\hline & Maropitant & $180,0 \pm 15 \mathrm{~A}$ & $152,5 \pm 18,7$ & $165,7 \pm 21,8 \mathrm{~B}$ & $209,3 \pm 22,3 \mathrm{~B}$ & $202,5 \pm$ & $216,8 \pm 20,0 \mathrm{~B}$ & $205,2 \pm 22,6 \mathrm{~B}$ \\
\hline & Morfina +maropitant & $170 \pm 13$ & $169,0 \pm 65,4 \mathrm{a}$ & $17,9 \mathrm{~B}$ & $198,5 \pm 20,6$ & $192,7 \pm 24,4$ & $184,8 \pm 50,9$ & $190,0 \pm 21,7$ \\
\hline \multirow{3}{*}{$\begin{array}{l}\text { Frequência respiratória, } \\
\text { mpm }\end{array}$} & Morfina & $39,0 \pm 15,6$ & $24,8 \pm 13,4$ & $20,8 \pm 15,8$ & $18,0 \pm 8,7$ & $17,6 \pm 9,2$ & $26,4 \pm 21,3$ & $21,6 \pm 14,9$ \\
\hline & Maropitant & $16,0 \pm 12,4$ & $17,3 \pm 3,3$ & $18,7 \pm 6,0$ & $19,3 \pm 3,9$ & $19,3 \pm 5,3$ & $18,0 \pm 5,5$ & $20,7 \pm 7,8$ \\
\hline & Morfina +maropitant & $20 \pm 15,6$ & $14,0 \pm 3,3$ & $15,3 \pm 3,0$ & $16,0 \pm 4,4$ & $16,7 \pm 5,3$ & $16,0 \pm 4,9$ & $13,3 \pm 4,1$ \\
\hline \multirow{3}{*}{$\begin{array}{l}\text { Saturação periférica de } \\
\text { Oxihemoglobina, } \%\end{array}$} & Morfina & $97,5 \pm 2,1$ & $95,0 \pm 4,4$ & $94,3 \pm 5,1$ & $96,0 \pm 1,7$ & $96,3 \pm 1,5$ & $95,3 \pm 2,1$ & $97,0 \pm 2,9$ \\
\hline & Maropitant & $99,0 \pm 1,1$ & $93,5 \pm 3,7$ & $94,0 \pm 3,6$ & $95,3 \pm 4,2$ & $93,8 \pm 4,7$ & $94,5 \pm 4,0$ & $93,5 \pm 3,2$ \\
\hline & Morfina +maropitant & $98 \pm 1,3$ & $93,7 \pm$ & $95,2 \pm 1,3$ & $97,0 \pm 2,3$ & $96,3 \pm 2,7$ & $95,5 \pm 4,3$ & $95,3 \pm 3,6$ \\
\hline \multirow{3}{*}{$\begin{array}{l}\text { Pressão arterial } \\
\text { Sistólica, mmHg }\end{array}$} & Morfina & $84 \pm 8,5 \mathrm{~A}$ & $67,3 \pm 19,2$ & $76,2 \pm 17,0 \mathrm{~B}$ & $124,3 \pm 22,3 \mathrm{~B}$ & $136,7 \pm 29,7 \mathrm{~B}$ & $142,3 \pm 38,3 \mathrm{~B}$ & $125,3 \pm 29,5 \mathrm{~B}$ \\
\hline & Maropitant & $90 \pm 6,2 \mathrm{~A}$ & $81,2 \pm 17,7$ & $95,7 \pm 14,1 \mathrm{~B}$ & $121,7 \pm 24,2 \mathrm{~B}$ & $143,5 \pm 31,8 \mathrm{~B}$ & $144 \pm 37 \mathrm{~B}$ & $123,7 \pm 25,4 \mathrm{~B}$ \\
\hline & Morfina +maropitant & $88 \pm 4,5 \mathrm{~A}$ & $88,3 \pm 40,9$ & $83,7 \pm 9,2 \mathrm{~B}$ & $140,7 \pm 28,6 \mathrm{~B}$ & $152 \pm 34,8 \mathrm{~B}$ & $167,2 \pm 18,4 \mathrm{~B}$ & $139,7 \pm 22,8$ \\
\hline \multirow{3}{*}{ Temperatura esofágica, ${ }^{\circ} \mathrm{C}$} & Morfina & $37,2 \pm 0,5$ & $36,9 \pm 0,9$ & $37,0 \pm 1,0$ & $36,5 \pm 1,2$ & $36,2 \pm 1,0$ & $36,2 \pm 1,3$ & $36,6 \pm 0,8$ \\
\hline & Maropitant & $38,6 \pm 0,6 \mathrm{~A}$ & $37,9 \pm 0,7$ & $37,4 \pm 0,8$ & $37,2 \pm 0,8$ & $37,1 \pm 0,8 \mathrm{~B}$ & $36,9 \pm 0,9 \mathrm{~B}$ & $36,6 \pm 1,1 \mathrm{~B}$ \\
\hline & Morfina +maropitant & $38,4 \pm 0,4$ & $37,2 \pm 0,3$ & $37,0 \pm 0,6$ & $36,8 \pm 0,6$ & $36,5 \pm 0,8$ & $36,7 \pm 0,6$ & $36,4 \pm 0,9$ \\
\hline
\end{tabular}

Letras maiúsculas nas linhas indicam diferença significativa entre momentos, dentro do mesmo grupo.

Quanto a temperatura corporal, não houve diferenças entre grupos, e nem dentro de um mesmo grupo. Entretanto, no grupo maropitant, 5 minutos após a aplicação dos tratamentos a temperatura se apresentou maior quando comparada ao momento após a sutura da musculatura e ao final da cirurgia, porém nenhum dos animais apresentou hipotermia ou hipertermia no período trans-operatório, com valores dentro dos limites de referência para a espécie (Cunningham, 2011).

O plano anestésico foi dividido em 1, 2 e 3, sendo eles superficial, ideal e profundo, respectivamente. $\mathrm{Na}$ estatística, não houve diferenças significativas entre grupos ou dentro de um mesmo grupo, isso pode ser explicado pelo cuidado do anestesista em manter o plano adequado, respeitando as taxas de administração dos fármacos.

Quanto às escalas utilizadas para mensuração de dor pós-operatória (multidimensional da dor aguda em felinos e analógica visual), não houve diferenças entre grupos, e realizando o somatório da pontuação de ambos os avaliadores por escala e por tempo, foi observado que para ambos os avaliadores a pontuação dos três grupos foi semelhante, e decresce para zero a partir de 12 horas pós-cirúrgicas (T12) (Figura 1). Ainda, pela figura 1, podemos observar a baixa pontuação atribuída pela EMADOR, o que pode estar relacionado a experiência do cirurgião, o que gera menor manipulação dos tecidos, pois segundo Mathews (2000), a duração e extensão do procedimento, o grau de manipulação de tecidos, idade e escore corporal do animal influenciam no grau de dor da OSH.

Comparando as duas escalas, houve uma maior sensibilidade na avaliação pela escala EMADOR, pois nenhum animal receberia resgate analgésico pela avaliação da EAV. Isso se deve ao fato de a metodologia utilizada permitir uma maior interação entre o avaliador e o animal, viabilizando um maior estímulo e percepção de movimentos e reações. O uso da escala EMADOR limita a subjetividade, e aumenta a precisão no reconhecimento, e na mensuração da dor específica em gatos, principalmente pela descrição detalhada de alterações comportamentais que são observadas (Brondani et al., 2011). De acordo com Brondani et al. (2012) a falta de um critério objetivo que oriente a avaliação, torna as escalas unidimensionais (EAV, escala numérica e escala descritiva simples) extremamente subjetivas e demasiadamente dependentes da experiência do observador.

De acordo com Hansen (2003), os animais podem ter uma limitação na expressão do comportamento normal por estarem presentes em um ambiente hospitalar e pela presença dos avaliadores. Segundo Mathews (2000), o animal nem sempre demonstra sinais claros de dor, sendo necessário reconhecer os sinais mais sutis. A familiarização com as reações a estímulos dolorosos, assim como com as diferentes 
personalidades dos pacientes é um ponto crucial nesse reconhecimento (Shaffran, 2008). Além da personalidade do animal e estímulos ambientais, outro fator a ser considerado é o avaliador, quanto sua experiência, convívio e conhecimento comportamental da espécie, aliado a sensibilidade em avaliar até os indícios mais suaves de desconforto. Vale salientar que os avaliadores eram ambos do gênero feminino, devidamente treinados, com o mesmo grau de experiência em estudos de nocicepção e manipulação com animais da espécie felina. Para amenizar o estresse do ambiente e da presença de avaliadores, a metodologia deste estudo optou por internar as gatas com 24 horas de antecedência ao procedimento cirúrgico. Sabe-se que existe variabilidade nos resultados quando mais do que um observador avalia o animal (Epstein, 2011; Hellyer et al., 2007; Holton et al., 1998) o que também propicia um super ou subdiagnóstico (Gaynor \& Muir, 2009).

Quanto às categorias da EMADOR, não houve diferenças entre grupos ou dentro de um mesmo grupo nos quesitos analisados, exceto a miscelânea, em que 2 horas pós-cirúrgicas (T2) apresentou-se maior quando comparada a 24 horas pós-procedimento (T24) no grupo morfina. No grupo maropitant, a pontuação em 4 horas pós-cirúrgicas demonstrou-se mais elevada quando comparada a 6 (T6) e 24 horas pós-cirúrgicas (T24), e, em 2 horas após o procedimento (T2), foi maior que 24 horas pós-cirúrgicas (T24). A miscelânea é um quesito avaliativo da EMADOR que abrange um ou mais comportamentos associados, subdivididos em quatro variáveis, dos quais o comportamento designado como: "o gato está contraindo e estendendo os membros pélvicos e/ou o gato está contraindo os músculos abdominais (flanco)." foi o mais pontuado em todos os grupos. Isso provavelmente se deve ao procedimento realizado, no qual há a manipulação de vísceras abdominais.

O resgate analgésico foi efetuado quando a pontuação da EMADOR foi igual ou superior a 8 para um ou ambos os avaliadores, e/ou quando pela EAV atingisse 3 pontos ou mais. Nota-se pela figura 2, que em todos os grupos houve necessidade de resgate já com 2 horas de pós-operatório, o que demonstra que nenhum protocolo utilizado é suficiente para abolir a dor desencadeada pelo procedimento de OSH. Além disso, pela curva de sobrevivência, não houve diferença significativa entre os grupos, mostrando que a possibilidade de receber resgate em todos os grupos foi igual.

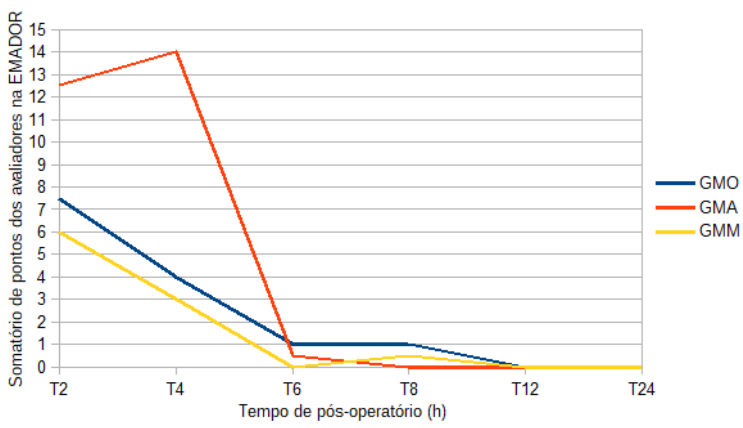

Figura 1. Somatório de pontos dos dois avaliadores pela escala multidimensional de dor aguda em felinos, em gatas submetidas a ovariohisterectomia eletiva e tratadas com morfina (GMO), maropitant (GMA) e morfina associada a maropitant (GMM).

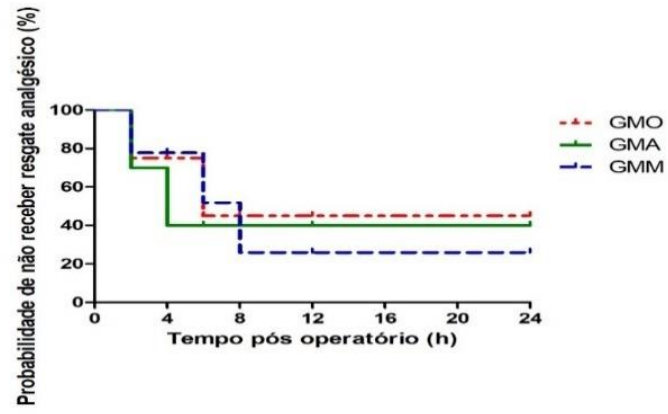

Figura 2. Análise de sobrevivência (curva de KaplanMeyer) de 18 gatas submetidas a OSH eletiva e pré-medicadas com maropitant (GMA), morfina (GMO) ou maropitant associado a morfina (GMM).

Em um estudo realizado por Druziani (2018) no qual foi comparado o efeito analgésico do maropitant ao da metadona e sua associação em cadelas, concluiu que não houve diferença na avaliação de dor no pós-operatório nem no número de resgates aplicados, sugerindo que a analgesia do maropitant isolado ou em associação a metadona foi semelhante ao da metadona. Já no estudo conduzido por Marquez et al. (2015) ao comparar maropitant à morfina, observaram efeito antinociceptivo superior do antagonista NK1 durante o período transoperatório de cadelas submetidas à OSH eletiva, com menores valores de frequência cardíaca, pressão arterial sistólica e requerimento de anestésico geral volátil. Os animais ainda tiveram menores escores de dor à extubação e aos 180 minutos, com retorno mais precoce do apetite, dado contrário ao achado neste estudo, em que não houve diferença estatística entre grupos em relação ao apetite dos animais durante 24 horas. 
Uma das dificuldades do estudo foi a mensuração da PAS pelos avaliadores, visto que esta é influenciada por fatores ambientais e comportamentais característicos dos animais presentes no estudo, levando a sua alteração ou impossibilidade de aferição, a qual ocorreu em 3 felinos, sendo estes um em cada grupo, onde os avaliadores foram impedidos de mensurar devido ao comportamento do animal. Sabe-se pela literatura que estímulos álgicos irão produzir alteração nos parâmetros fisiológicos, sendo importantes para o processo de avaliação da dor (Hellyer et al., 2007). No entanto, de acordo com Brondani et al. (2011), cada aspecto da escala pode ser avaliado de maneira isolada, o que possibilita na ausência de determinado recurso para avaliação de itens presentes na escala, ser omitido, sem interferir nos resultados.

\section{Referências}

Anderline, G. A. (2007). Benefícios do envolvimento do animal de companhia (cão e gato), na terapia, na socialização e bem estar das pessoas e o papel do médico veterinário. Revista Do Conselho Federal de Medicina Veterinária, 13(41), 70-75.

Beaver, B. V. (2005). Comportamento Felino-Um Guia para Veterinários. Editora Roca.

Brondani, J T, Luna, S. P. L., Minto, B. W., Santos, B. P. R., Beier, S. L., Matsubara, L. M., \& Padovani, C. R. (2012). Validity and responsiveness of a multidimensional composite scale to assess postoperative pain in cats. Arquivo Brasileiro de Medicina Veterinária e Zootecnia, 64(6), 15291538.

Brondani, J. T., Luna, S. P. L. \& Padovani, C. R. (2011). Refinement and initial validation of a multidimensional composite scale for use in assessing acute postoperative pain in cats. American Journal of Veterinary Research, 72(2), 174-183. https://doi.org/10.2460/ajvr.72.2.174

Corrêa, J. M. X., Soares, P. C. L. R., Niella, R. V, Costa, B. A., Ferreira, M. S., Junior, A. C. S., Sena, A. S., Sampaio, K. M. O. R., Silva, E. B., \& Silva, F. L. (2019). Evaluation of the antinociceptive effect of maropitant, a neurokinin-1 receptor antagonist, in cats undergoing ovariohysterectomy. Veterinary Medicine International, 2019. https://doi.org/10.1155/2019/9352528.

Cunningham, J. (2011). Tratado de fisiologia veterinária. Guanabara Koogan.

de la Puente-Redondo, V., Tingley, F. D., Schneider, R. P., \& Hickman, M. A. (2007). The neurokinin1 antagonist activity of maropitant, an antiemetic drug for dogs, in a gerbil model. Journal of Veterinary Pharmacology and Therapeutics, 30(4), 281-287. https://doi.org/10.1111/j.13652885.2007.00847.x.

Deschamps, K., \& Couture, R. (2005). The ventral tegmental area as a putative target for tachykinins in cardiovascular regulation. British Journal of Pharmacology, 145(6), 712-727. https://doi.org/10.1038/sj.bjp.0706249.

Druziani, J. T. (2018). Avaliação do potencial analgésico do Maropitant em cadelas submetidas à ovariohisterectomia eletiva.

Epstein, M. E. (2011). Recognition, assessment and scoring of pain in dogs and cats (Proceedings). North American Veterinary Conference, 9-15.

Fantoni, D. T., Mastrocinque, S., Fantoni, D. T., \& Cottopassi, S. R. (2002). Fisiopatologia e controle da dor. In D. T. Fabntoni (Ed.), Anestesia em cães e gatos (pp. 323-336). Roca.

Garcia-Recio, S., \& Gascón, P. (2015). Biological and pharmacological aspects of the NK1-receptor. BioMed Research International, 2015, 1-15. https://doi.org/10.1155/2015/495704.

Gaynor, J. S., \& Muir, W. W. (2009). Manual de controle da dor em medicina veterinária (Vol. 1). MedVet.

Hansen, B. D. (2003). Assessment of pain in dogs: veterinary clinical studies. ILAR Journal, 44(3), 197205. https://doi.org/10.1093/ilar.44.3.197.

Hellebrekers, L. J. (2002). Dor em animais: uma abordagem com orientação prática para um controle eficaz da dor em animais. Manole.

Hellyer, P., Rodan, I., Brunt, J., Downing, R., Hagedorn, J. E., \& Robertson, S. A. (2007). AAHA/AAFP pain management guidelines for dogs and cats. Journal of Feline Medicine \& Surgery, 9(6), 466480. https://doi.org/10.5326/JAAHA-MS-7331. 
Hellyer, P. W. (1997). Management of acute and surgical pain. Seminars in Veterinary Medicine and Surgery (Small Animal), 12(2), 106-114.

Holton, L. L., Scott, E. M., Nolan, A. M., Reid, J., Welsh, E., \& Flaherty, D. (1998). Comparison of three methods used for assessment of pain in dogs. Journal of the American Veterinary Medical Association, 212(1), 61-66.

Liehmann, L., Mosing, M., \& Auer, U. (2006). A comparison of cardiorespiratory variables during isoflurane-fentanyl and propofol-fentanyl anaesthesia for surgery in injured cats. Veterinary Anaesthesia and Analgesia, 33(3), 158-168. https://doi.org/10.1111/j.1467-2995.2005.00251.x.

Marquez, M., Boscan, P., Weir, H., Vogel, P., \& Twedt, D. C. (2015). Comparison of nk-1 receptor antagonist (maropitant) to morphine as a pre-anaesthetic agent for canine ovariohysterectomy. PLoS One, 10(10), e0140734. https://doi.org/10.1371/journal.pone.0140734.

Mastrocinque, S. (2005). Avaliação do emprego do tramadol epidural ou sistêmico e da morfina epidural em cadelas submetidas à ovariohisterectomia. Universidade de São Paulo.

Mathews, K. A. (2000). Pain assessment and general approach to management. Veterinary Clinics: Small Animal Practice, 30(4), 729-755. https://doi.org/10.1016/S0195-5616(08)70004-4.

Shaffran, N. (2008). Pain management: the veterinary technician's perspective. Veterinary Clinics of North America: Small Animal Practice, 38(6), 1415-1428.

Short, C. E. (1998). Fundamentals of pain perception in animals. Applied Animal Behaviour Science, 59(1-3), 125-133. https://doi.org/10.1016/S0168-1591(98)00127-0.

Silva, F. L. (2011). Terapêutica da dor na cirurgia de cães e gatos: revisão. Revista Veterinária Em Foco, 9(1).

Spinosa, H. de S., Górniak, S. L., \& Bernardi, M. M. (2006). Farmacologia aplicada à medicina veterinária. Koogan Guanabara.

Swallow, A., Rioja, E., Elmer, T., \& Dugdale, A. (2017). The effect of maropitant on intraoperative isoflurane requirements and postoperative nausea and vomiting in dogs: a randomized clinical trial. Veterinary Anaesthesia and Analgesia, 44(4), 785-793. https://doi.org/10.1016/j.vaa.2016.10.006.

Teixeira, M. J., \& Figueiró, J. B. (2001). Dor - epidemiologia e evolução histórica da dor. Moreira Júnior.

Histórico do artigo:

Recebido: 20 de agosto de 2020 . Aprovado: 14 de outubro de 2020. Disponível online: 18 de janeiro de 2021.
Licenciamento: Este artigo é publicado na modalidade Acesso Aberto sob a licença Creative Commons Atribuição 4.0 (CC-BY 4.0), a qual permite uso irrestrito, distribuição, reprodução em qualquer meio, desde que o autor e a fonte sejam devidamente creditados. 